\title{
Thin-shell wormholes from the regular Hayward black hole
}

\author{
M. Halilsoy ${ }^{\mathrm{a}}$, A. Ovgun ${ }^{\mathrm{b}}$, S. Habib Mazharimousavi ${ }^{\mathrm{c}}$ \\ Department of Physics, Eastern Mediterranean University, G. Magusa, North Cyprus, Mersin 10, Turkey
}

Received: 21 January 2014 / Accepted: 24 February 2014 / Published online: 13 March 2014

(C) The Author(s) 2014. This article is published with open access at Springerlink.com

\begin{abstract}
We revisit the regular black hole found by Hayward in 4-dimensional static, spherically symmetric spacetime. To find a possible source for such a spacetime we resort to the nonlinear electrodynamics in general relativity. It is found that a magnetic field within this context gives rise to the regular Hayward black hole. By employing such a regular black hole we construct a thin-shell wormhole for the case of various equations of state on the shell. We abbreviate a general equation of state by $p=\psi(\sigma)$ where $p$ is the surface pressure which is a function of the mass density $(\sigma)$. In particular, linear, logarithmic, Chaplygin, etc. forms of equations of state are considered. In each case we study the stability of the thin shell against linear perturbations. We plot the stability regions by tuning the parameters of the theory. It is observed that the role of the Hayward parameter is to make the TSW more stable. Perturbations of the throat with small velocity condition are also studied. The matter of our TSWs, however, remains exotic.
\end{abstract}

\section{Introduction}

Thin-shell wormholes (TSWs) constitute one of the wormhole classes in which the exotic matter is confined on a hypersurface and therefore can be minimized [1-16] (the $d$-dimensional thin-shell wormhole is considered in [17] and the case with a cosmological constant is studied in [18]). Finding a physical (i.e. non-exotic) source to wormholes of any kind remains as ever a challenging problem in Einstein's general relativity. In this regard we must add that modified theories of gravity present more alternatives with their extra degrees of freedom. We recall, however, that each modified theory partly cures things, while it partly adds its own complications. Staying within Einstein's general relativity and

\footnotetext{
a e-mail: mustafa.halilsoy@emu.edu.tr

b e-mail: ali.ovgun@emu.edu.tr

c e-mail: habib.mazhari@emu.edu.tr
}

finding remedies seems to be the prominent approach, provided the proper spacetimes are employed. An interesting class of spacetimes that may serve the purpose is the spacetimes of regular black holes.

Our motivation for choosing a regular black hole in the wormhole construction can be justified by the fact that a regular system can be established from a finite energy. In high energy collision experiments for instance, the formation of such regular objects is more tenable. Such a black hole was discovered first by Bardeen and came to be known as Bardeen black hole [19-22]. Ayon-Beato and Garcia in [22] introduced a nonlinear electric field source for the Bardeen black hole. Bronnikov, later on, showed that the regular 'electric' black hole, e.g., the one considered by Ayon-Beato and Garcia, is not a quite correct solution to the field equations, because in these solutions the electromagnetic Lagrangian is inevitably different in different parts of space. On the contrary, quite correct solutions of this kind (and even with the same metric) can be readily obtained with a magnetic field (since in nonlinear electrodynamics (NED) there is no such duality as in the linear Maxwell theory). All this is described in detail in [23-25]. A similar type of black hole solution was given by Hayward [26], which provides the main motivation and fuel to the present study. This particular black hole solution has well-defined asymptotic limits, namely it is Schwarzschild for $r \rightarrow \infty$ and de Sitter for $r \rightarrow 0$. In order to make a better account of the Hayward black hole we attempt first to explore its physical source. For this reason we search for the NED and find that a magnetic field within this theory accounts for such a source. Note that every NED does not admit a linear Maxwell limit and indeed this is precisely the case that we face in the present problem. In other words, if our NED model did have a Maxwell limit, then the Hayward spacetime should coincide with the ReissnerNordström (RN) limit. Such a limit does not exist in the present problem. Once we fix our bulk spacetime the next step is to locate the thin shell which must lie outside the event horizon of the black hole. The surface energy-momentum 
tensor on the shell must satisfy the Israel junction conditions [27-31]. As the Equation of State (EoS) for the energymomentum on the shell we choose different models, which are abbreviated by $p=\psi(\sigma)$. Here $p$ stands for the surface pressure, $\sigma$ is the mass (energy) density and $\psi(\sigma)$ is a function of $\sigma$. We consider the following cases: (1) linear gas (LG) [32,33], where $\psi(\sigma)$ is a linear function of $\sigma$; (2) Chaplygin gas (CG) [34,35], where $\psi(\sigma) \sim \frac{1}{\sigma}$; (3) generalized Chaplygin gas (GCG) [36-40], where $\psi(\sigma) \sim \frac{1}{\sigma^{v}}(\nu=$ constant); (4) modified generalized Chaplygin gas (MGCG) [41-44], where $\psi(\sigma) \sim \mathrm{LG}+\mathrm{GCG}$; and (5) logarithmic gas $(\operatorname{LogG})$, where $\psi(\sigma) \sim \ln |\sigma|$.

For each of the cases we plot the second derivative of the derived potential function $V^{\prime \prime}\left(a_{0}\right)$, where $a_{0}$ stands for the equilibrium point. The region where the second derivative is positive (i.e. $\left.V^{\prime \prime}\left(a_{0}\right)>0\right)$ yields the regions of stability which are all depicted in figures. This summarizes the strategy that we adopt in the present paper for the stability of the thin-shell wormholes constructed from the Hayward black hole.

The organization of the paper is as follows. Section 2 reviews the Hayward black hole and determines a Lagrangian for it. A derivation of the stability condition is carried out in Sect. 3. Particular examples of the equation of state follow in Sect. 4. Small velocity perturbations are the subject of Sect. 5. The paper ends with the our conclusion in Sect. 6.

\section{Regular Hayward black hole}

The spherically symmetric static Hayward nonsingular black hole introduced in [26] is given by the following line element:

$$
\begin{aligned}
\mathrm{d} s^{2}= & -\left(1-\frac{2 m r^{2}}{r^{3}+2 m l^{2}}\right) \mathrm{d} t^{2} \\
& +\left(1-\frac{2 m r^{2}}{r^{3}+2 m l^{2}}\right)^{-1} \mathrm{~d} r^{2}+r^{2} \mathrm{~d} \Omega^{2}
\end{aligned}
$$

in which $m$ and $l$ are two free parameters and

$$
\mathrm{d} \Omega^{2}=\mathrm{d} \theta^{2}+\sin ^{2} \theta \mathrm{d} \phi^{2}
$$

The metric function of this black hole $f(r)=\left(1-\frac{2 m r^{2}}{r^{3}+2 m l^{2}}\right)$ at large $r$ behaves as

$\lim _{r \rightarrow \infty} f(r) \rightarrow 1-\frac{2 m}{r}+\mathcal{O}\left(\frac{1}{r^{4}}\right)$,

while at small $r$

$$
\lim _{r \rightarrow 0} f(r) \rightarrow 1-\frac{r^{2}}{l^{2}}+\mathcal{O}\left(r^{5}\right) .
$$

From the asymptotic form of the metric function at small and large $r$ one observes that the Hayward nonsingular black hole is a de Sitter black hole for small $r$ and Schwarzschild spacetime for large $r$. The curvature scalars are all finite at $r=0$ [45]. The Hayward black hole admits an event horizon, which is the largest real root of the following equation:

$r^{3}-2 m r^{2}+2 m l^{2}=0$

Setting $r=m \rho$ and $l=m \lambda$ this becomes

$\rho^{3}-2 \rho^{2}+2 \lambda^{2}=0$

which admits no horizon (regular particle solution) for $\lambda^{2}>\frac{16}{27}$, a single horizon (regular extremal black hole) for $\lambda^{2}=\frac{16}{27}$, and double horizons (regular black hole with two horizons) for $\lambda^{2}<\frac{16}{27}$. Therefore the important parameter is the ratio $\frac{l}{m}$ with critical ratio at $\left(\frac{l}{m}\right)_{\text {crit. }}=\frac{4}{3 \sqrt{3}}$, but not $l$ and $m$ separately. This suggests setting $m=1$ in the sequel without loss of generality, i.e., $f(r)=1-\frac{2 r^{2}}{r^{3}+2 l^{2}}$. Accordingly for $l^{2}<\frac{16}{27}$ the event horizon is given by

$r_{h}=\frac{1}{3}\left(\sqrt[3]{\Delta}+\frac{4}{\sqrt[3]{\Delta}}+2\right)$

in which $\Delta=8-27 l^{2}+3 \sqrt{27 l^{2}\left(3 l^{2}-2\right)}$. For the case of an extremal black hole, i.e., $l^{2}=\frac{16}{27}$, the single horizon occurs at $r_{h}=\frac{4}{3}$. For the case $l^{2} \leq \frac{16}{27}$ the standard Hawking temperature at the event horizon is given by

$T_{H}=\frac{f^{\prime}\left(r_{h}\right)}{4 \pi}=\frac{1}{4 \pi}\left(\frac{3}{2}-\frac{2}{r_{h}}\right)$,

which clearly for $l^{2}=\frac{16}{27}$ vanishes and for $l^{2}<\frac{16}{27}$ is positive (one should note that $r_{h} \geq \frac{4}{3}$ ). Considering the standard definition for the entropy of the black hole $S=\frac{\mathcal{A}}{4}$, in which $\mathcal{A}=4 \pi r_{h}^{2}$, one finds the heat capacity of the black hole defined by

$C_{l}=\left(T_{H} \frac{\partial S}{\partial T_{H}}\right)$

and determined as

$C_{l}=4 \pi r_{h}^{3}\left(\frac{3}{2}-\frac{2}{r_{h}}\right)$,

which is clearly non-negative. The fact that $C_{l}>0$ shows that thermodynamically the black hole is stable. 
2.1 Magnetic monopole field as a source for the Hayward black hole

We consider the action

$\mathcal{I}=\frac{1}{16 \pi} \int d^{4} x \sqrt{-g}(R-\mathcal{L}(F))$,

in which $R$ is the Ricci scalar and

$\mathcal{L}(F)=-\frac{24 m^{2} l^{2}}{\left[\left(\frac{2 P^{2}}{F}\right)^{3 / 4}+2 m l^{2}\right]^{2}}=-\frac{6}{l^{2}\left[1+\left(\frac{\beta}{F}\right)^{3 / 4}\right]^{2}}$

is the nonlinear magnetic field Lagrangian density with $F=$ $F_{\mu \nu} F^{\mu \nu}$, the Maxwell invariant with $l$ and $\beta$, two constant positive parameters. Let us note that the subsequent analysis will fix $\beta$ in terms of the other parameters. The magnetic field two-form is given by

$\mathbf{F}=P \sin ^{2} \theta \mathrm{d} \theta \wedge \mathrm{d} \phi$

in which $P$ stands for the magnetic monopole charge. This field form together with the line element (1) implies

$F=\frac{2 P^{2}}{r^{4}}$

The Einstein-NED field equations are $(8 \pi G=c=1)$

$G_{\mu}^{v}=T_{\mu}^{v}$

in which

$T_{\mu}^{v}=-\frac{1}{2}\left(\mathcal{L} \delta_{\mu}^{v}-4 F_{\mu \lambda} F^{\lambda v} \mathcal{L}_{F}\right)$

with $\mathcal{L}_{F}=\frac{\partial \mathcal{L}}{\partial F}$. One can show that using $\mathcal{L}(F)$ given in (12), the Einstein equations admit the Hayward regular black hole metric provided $\beta=\frac{2 P^{2}}{\left(2 m l^{2}\right)^{4 / 3}}$. The weak field limit of the Lagrangian (12) can be found by expanding the Lagrangian about $F=0$, which leads to

$\mathcal{L}(F)=-\frac{6 F^{3 / 2}}{l^{2} \beta^{3 / 2}}+\frac{12 F^{9 / 4}}{l^{2} \beta^{9 / 4}}+\mathcal{O}\left(F^{3}\right)$.

It is observed that in the weak field limit the NED Lagrangian does not yield the linear Maxwell Lagrangian, i.e., $\lim _{F \rightarrow 0} \mathcal{L}(F) \neq-F$. For this reason we do not expect that the metric function in the weak field limit gives the RN black hole solution as described in (3).

\section{Stable thin-shell wormhole condition}

In this section we use the standard method of making a timelike TSW and for this reason we consider a timelike thin shell located at $r=a\left(a>r_{h}\right)$ by cutting the region $r<a$ from the Hayward regular black hole and pasting two copies of it at $r=a$. On the shell the spacetime is chosen to be

$\mathrm{d} s^{2}=-\mathrm{d} \tau^{2}+a(\tau)^{2}\left(\mathrm{~d} \theta^{2}+\sin ^{2} \theta \mathrm{d} \phi^{2}\right)$

in which $\tau$ is the proper time on the shell. To make a consistent 2+1-dimensional timelike shell at the intersection the two 3+ 1-dimensional hypersurfaces we have to fulfill the Lanczos conditions [27-31]. These are the Einstein equations on the shell,

$\left[K_{i}^{j}\right]-[K] \delta_{i}^{j}=-S_{i}^{j}$

in which a bracket of $X$ is defined as $[X]=X_{2}-X_{1}, K_{i}^{j}$ is the extrinsic curvature tensor in each part of the thin shell, and $K$ denotes its trace. $S_{i}^{j}$ is the energy momentum tensor on the shell such that $S_{\tau}^{\tau}=-\sigma$ stands for the energy density and $S_{\theta}^{\theta}=p=S_{\phi}^{\phi}$ are the surface pressures. One can explicitly find

$\sigma=-\frac{4}{a} \sqrt{f(a)+\dot{a}^{2}}$

and

$p=2\left(\frac{\sqrt{f(a)+\dot{a}^{2}}}{a}+\frac{\ddot{a}+f^{\prime}(a) / 2}{\sqrt{f(a)+\dot{a}^{2}}}\right)$.

Consequently the energy and pressure densities in a static configuration at $a=a_{0}$ are given by

$\sigma_{0}=-\frac{4}{a_{0}} \sqrt{f\left(a_{0}\right)}$

and

$p_{0}=2\left(\frac{\sqrt{f\left(a_{0}\right)}}{a_{0}}+\frac{f^{\prime}\left(a_{0}\right) / 2}{\sqrt{f\left(a_{0}\right)}}\right)$.

To investigate the stability of such a wormhole we apply a linear perturbation in which the EoS

$p=\psi(\sigma)$

with an arbitrary equation for $\psi(\sigma)$ is adopted for the thin shell. In addition to this relation between $p$ and $\sigma$ the energy conservation identity also imposes

$S_{; j}^{i j}=0$ 
which in closed form amounts to

$S_{, j}^{i j}+S^{k j} \Gamma_{k j}^{i}+S^{i k} \Gamma_{k j}^{j}=0$,

or equivalently, after the line element (18),

$\frac{\partial}{\partial \tau}\left(\sigma a^{2}\right)+p \frac{\partial}{\partial \tau}\left(a^{2}\right)=0$.

This equation can be rewritten as

$\dot{a}^{2}+V(a)=0$

where $V(a)$ is given by

$V(a)=f-\left(\frac{a \sigma}{4}\right)^{4}$

and $\sigma$ is the energy density after the perturbation. Equation (28) is a 1-dimensional equation of motion in which the oscillatory motion for $a$ in terms of $\tau$ about $a=a_{0}$ is a consequence of having $a=a_{0}$, the equilibrium point, which means $V^{\prime}\left(a_{0}\right)=0$ and $V^{\prime \prime}\left(a_{0}\right) \geq 0$. In the sequel we consider $f_{1}\left(a_{0}\right)=f_{2}\left(a_{0}\right)$, and therefore at $a=a_{0}$, one finds $V_{0}=V_{0}^{\prime}=0$. To investigate $V^{\prime \prime}\left(a_{0}\right) \geq 0$ we use the given $p=\psi(\sigma)$ to find

$\sigma^{\prime}\left(=\frac{\mathrm{d} \sigma}{\mathrm{d} a}\right)=-\frac{2}{a}(\sigma+\psi)$

and

$\sigma^{\prime \prime}=\frac{2}{a^{2}}(\sigma+\psi)\left(3+2 \psi^{\prime}\right)$

with $\psi^{\prime}=\frac{\mathrm{d} \psi}{\mathrm{d} \sigma}$. Finally

$V^{\prime \prime}\left(a_{0}\right)=f_{0}^{\prime \prime}-\frac{1}{8}\left[\left(\sigma_{0}+2 p_{0}\right)^{2}+2 \sigma_{0}\left(\sigma_{0}+p_{0}\right)\left(1+2 \psi^{\prime}\left(\sigma_{0}\right)\right)\right]$

where we have used $\psi_{0}=p_{0}$.

\section{Some models of exotic matter supporting the TSW}

Recently two of us analyzed the effect of the Gauss-Bonnet parameter on the stability of TSW in higher-dimensional EGB gravity [46]. In that paper some specific models of matter have been considered such as LG, CG, GCG, MGCG, and LogG. In this work we get closely to the same EoSs and we analyze the effect of Hayward's parameter in the stability of the TSW constructed above.
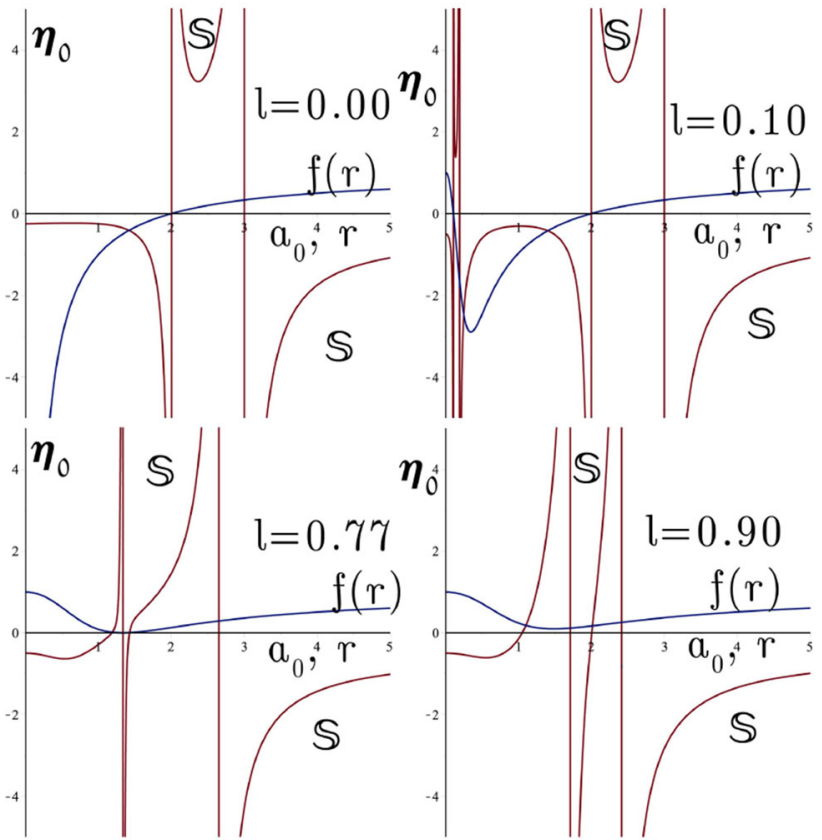

Fig. 1 Stability of TSW supported by LG in terms of $a_{0}$ and $\eta_{0}$ for $\ell=0.00,0.10,0.77$, and 0.90 . The value of $m=1$. The effect of Hayward's constant is to increase the stability of the TSW. We note that the stable regions are shown by $\mathcal{S}$ and the metric function is plotted too

\subsection{Linear gas (LG)}

In the case of a linear EoS, i.e.,

$\psi=\eta_{0}\left(\sigma-\sigma_{0}\right)+p_{0}$

in which $\eta_{0}$ is a constant parameter, one finds $\psi^{\prime}\left(\sigma_{0}\right)=\eta_{0}$. Figure 1 displays the region of stability in terms of $\eta_{0}$ and $a_{0}$ for different values of Hayward's parameter.

\subsection{Chaplygin gas (CG)}

For Chaplygin gas (CG) the EoS is given by

$\psi=\eta_{0}\left(\frac{1}{\sigma}-\frac{1}{\sigma_{0}}\right)+p_{0}$

where $\eta_{0}$ is a constant parameter, and which implies $\psi^{\prime}\left(\sigma_{0}\right)=-\frac{\eta_{0}}{\sigma_{0}^{2}}$. In Fig. 2 we plot the stability region in terms of $\eta_{0}$ and $a_{0}$ for different values of $\ell$.

\subsection{Generalized Chaplygin gas (GCG)}

The EoS of the Generalized Chaplygin gas can be cast into the form

$\psi(\sigma)=\eta_{0}\left(\frac{1}{\sigma^{v}}-\frac{1}{\sigma_{0}^{\nu}}\right)+p_{0}$ 


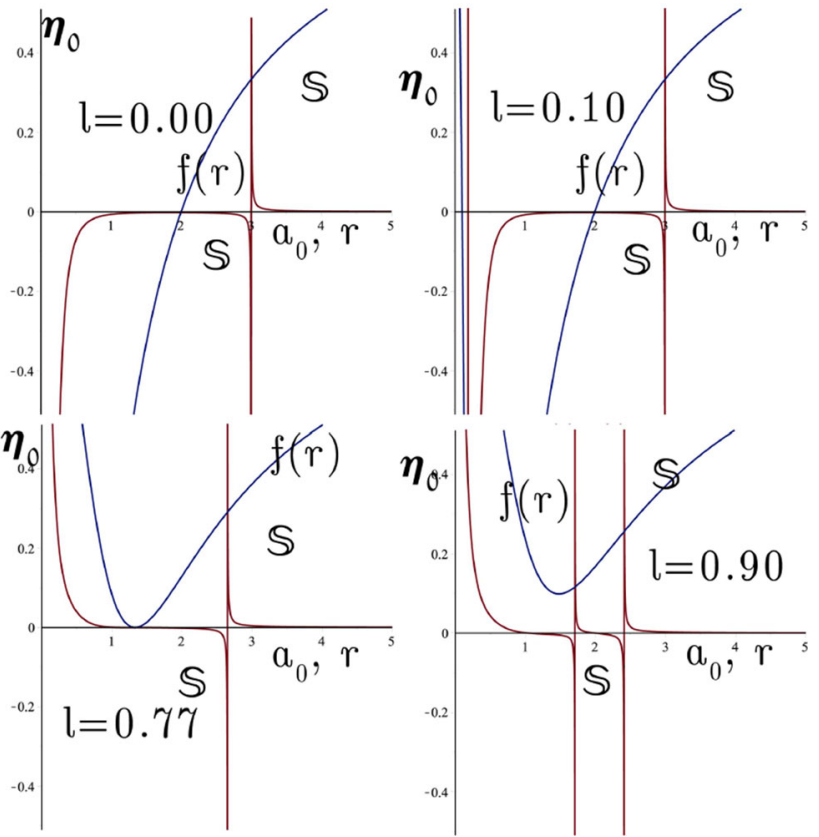

Fig. 2 Stability of TSW supported by CG in terms of $a_{0}$ and $\eta_{0}$ for $\ell=0.00,0.10,0.77$ and 0.90 . The value of $m=1$. The effect of Hayward's constant is to increase the stability of the TSW. We also plot the metric function to compare the horizon of the black hole and the location of the throat

in which $v$ and $\eta_{0}$ are constants. To see the effect of parameter $v$ on the stability we set the constant $\eta_{0}$ such that $\psi$ becomes

$\psi(\sigma)=p_{0}\left(\frac{\sigma_{0}}{\sigma}\right)^{v}$.

We find $\psi^{\prime}\left(\sigma_{0}\right)=-\frac{p_{0}}{\sigma_{0}} v$ and in Fig. 3 we plot the stability regions of the TSW supported by a GCG in terms of $v$ and $a_{0}$ with various values of $\ell$.

\subsection{Modified generalized Chaplygin gas (MGCG)}

A more general form of $\mathrm{CG}$ is called the modified generalized Chaplygin gas (MGCG), which is given by

$\psi(\sigma)=\xi_{0}\left(\sigma-\sigma_{0}\right)-\eta_{0}\left(\frac{1}{\sigma^{\nu}}-\frac{1}{\sigma_{0}^{\nu}}\right)+p_{0}$

in which $\xi_{0}, \eta_{0}$, and $v$ are free parameters. One then finds

$\psi^{\prime}\left(\sigma_{0}\right)=\xi_{0}+\eta_{0} \frac{\eta_{0} v}{\sigma_{0}^{v+1}}$.

To proceed we set $\xi_{0}=1$ and $v=1$ and in Fig. 4 we show the stability regions in terms of $\eta_{0}$ and $a_{0}$ with various values of $\ell$.
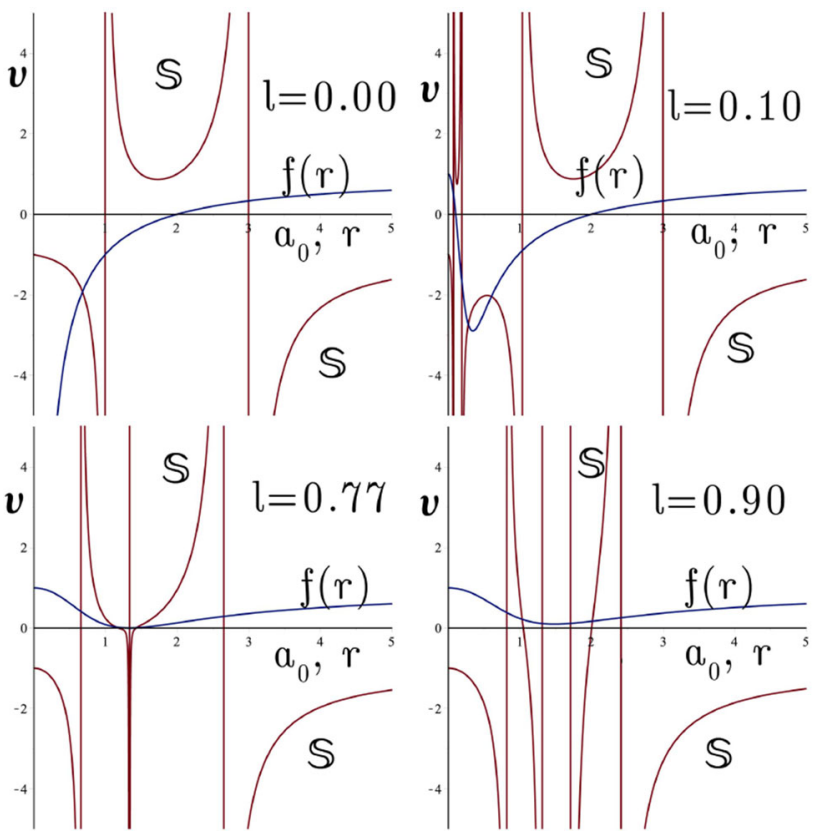

Fig. 3 Stability of TSW supported by GCG in terms of $a_{0}$ and $v$ for $\ell=0.00,0.10,0.77$ and 0.90 . The value of $m=1$. The effect of Hayward's constant is to increase the stability of the TSW. We also plot the metric function to compare the horizon of the black hole and the location of the throat
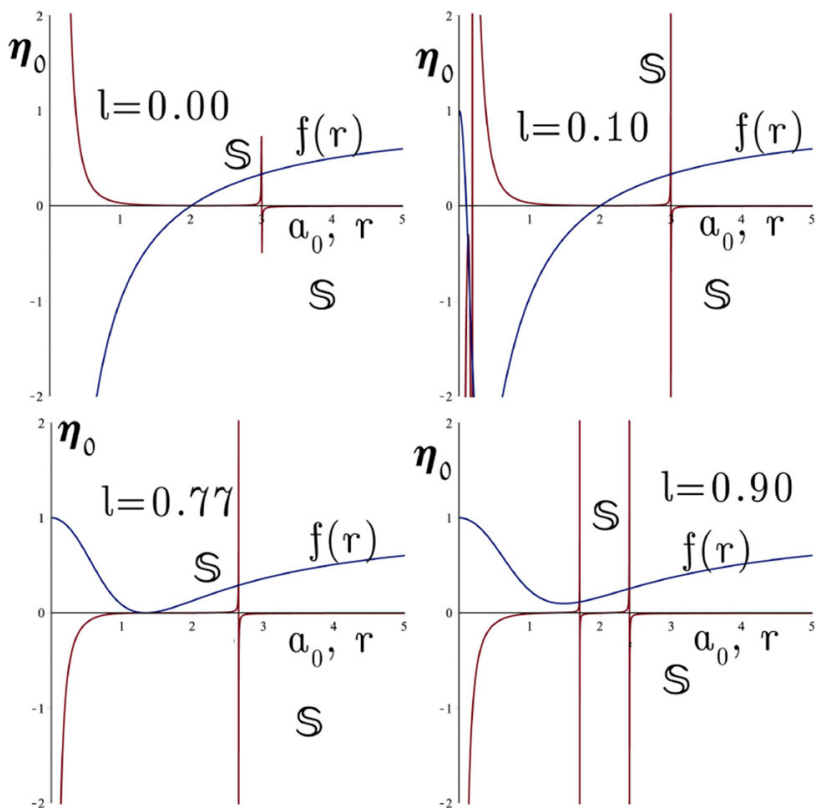

Fig. 4 Stability of TSW supported by MGCG in terms of $a_{0}$ and $\eta_{0}$ for $\ell=0.00,0.10,0.77$ and 0.90 . The value of $m=1$ and $\xi_{0}=\eta_{0}=1$. The effect of Hayward's constant is to increase the stability of the TSW. We also plot the metric function to compare the horizon of the black hole and the location of the throat 


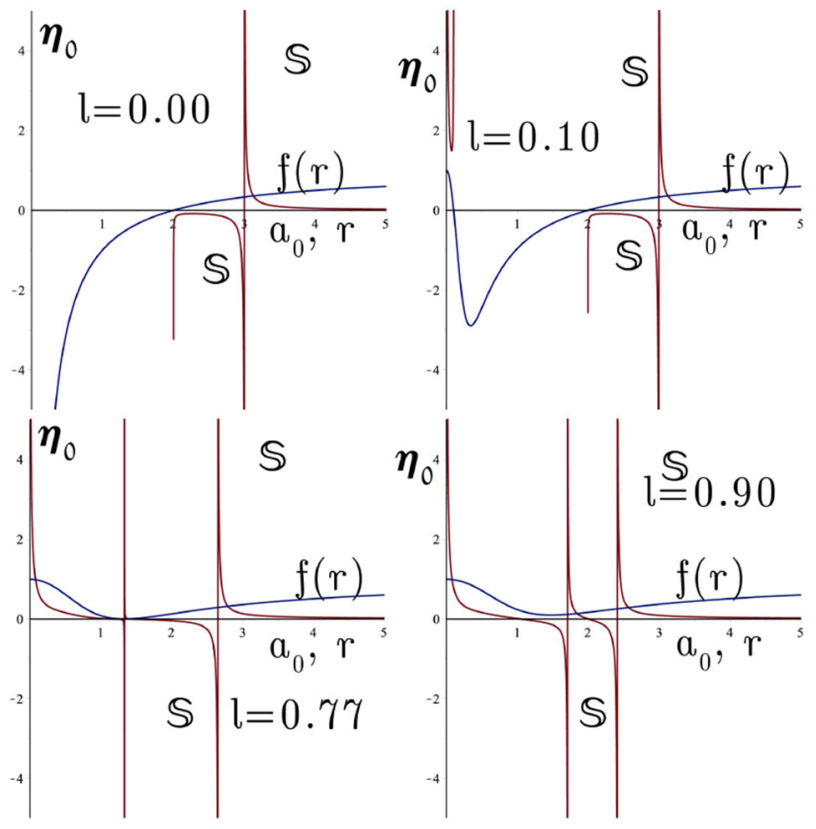

Fig. 5 Stability of TSW supported by LogG in terms of $a_{0}$ and $\eta_{0}$ for $\ell=0.00,0.10,0.77$ and 0.90 . The value of $m=1$. The effect of Hayward's constant is to increase the stability of the TSW. We also plot the metric function to compare the horizon of the black hole and the location of the throat

\subsection{Logarithmic gas ( $\log G)$}

In our last example we consider the logarithmic gas ( $\log G)$ given by

$\psi(\sigma)=\eta_{0} \ln \left|\frac{\sigma}{\sigma_{0}}\right|+p_{0}$

in which $\eta_{0}$ is a constant. For $\log G$ one finds

$\psi^{\prime}\left(\sigma_{0}\right)=\frac{\eta_{0}}{\sigma_{0}}$.

In Fig. 5 we plot the stability region for the TSW supported by $\log G$ and the effect of Hayward's parameter is shown clearly.

\section{Stability analysis for small velocity perturbations around the static solution}

In this section we restrict ourselves to the small velocity perturbations about the equilibrium point $a=a_{0}$, such that at any proper time after the perturbation we can consider the fluid supporting the shell to be approximately static. Thus one can accept the dynamic EoS of the wormhole to be the same as the static EoS [49-55]. This assumption, therefore, implies that the EoS is uniquely determined by $f(a)$ and $a$, described by (22) and (23), i.e.,

$p=-\frac{1}{2}\left(1+\frac{a f^{\prime}(a)}{2 f(a)}\right) \sigma$.

With this EoS together with (20) and (21) one finds the 1dimensional motion of the throat given by

$\ddot{a}-\frac{f^{\prime}}{2 f} \dot{a}^{2}=0$.

Now, an integration from both sides implies

$\dot{a}=\dot{a}_{0} \frac{\sqrt{f}}{\sqrt{f_{0}}}$

and a second integration gives

$\int_{a 0}^{a} \frac{\mathrm{d} a}{\sqrt{f(a)}}=\frac{\dot{a}_{0}}{\sqrt{f_{0}}}\left(\tau-\tau_{0}\right)$.

Note that $\dot{a}_{0}$ for the equilibrium point is zero but here after perturbation we assume that the perturbation consists of an initial small velocity which we call $\dot{a}_{0}$.

\subsection{The Schwarzschild example}

The last integral (44) depends on the bulk metric, so that it gives different results for different spacetimes. For the Schwarzschild bulk, we have $f(a)=1-\frac{2 m}{a}$, which on substitution in (44) yields

$$
\frac{\dot{a}_{0}}{\sqrt{f_{0}}}\left(\tau-\tau_{0}\right)=a \sqrt{f}-a_{0} \sqrt{f_{0}}+m \ln \left(\frac{a-m+a \sqrt{f}}{a_{0}-m+a_{0} \sqrt{f_{0}}}\right) .
$$

This motion is clearly not oscillatory, which indicates that the throat is unstable against a small velocity perturbation.

\subsection{The Hayward example}

For the case of the Hayward bulk spacetime, (44), up to the second order of $\ell$ leads to

$$
\begin{aligned}
& \frac{\dot{a}_{0}}{\sqrt{f_{0}}}\left(\tau-\tau_{0}\right) \tilde{=} a \sqrt{f}-a_{0} \sqrt{f_{0}} \\
& \quad+m \ln \left(\frac{a-m+a \sqrt{f}}{a_{0}-m+a_{0} \sqrt{f_{0}}}\right) \\
& \quad+2 \ell^{2}\left(\frac{2 a^{2}-2 a m-m^{2}}{3 m a^{2} \sqrt{f}}-\frac{2 a_{0}^{2}-2 a_{0} m-m^{2}}{3 m a_{0}^{2} \sqrt{f_{0}}}\right) .
\end{aligned}
$$

Similar to the previous case, this motion is not oscillatory, which implies that the throat is unstable against the 
small velocity perturbation. Nevertheless, (42) shows that the acceleration of the throat is given by $\ddot{a}=\frac{f^{\prime}}{2 f} \dot{a}^{2}$, which is positive for both the Schwarzschild and the Hayward bulk. Thus the motion of the throat is not oscillatory and consequently the corresponding TSW is not stable.

\section{Conclusion}

Thin-shell wormholes are constructed from the regular black hole (or non-black hole for a certain range of parameters) discovered by Hayward. We show first that this solution is powered by a magnetic monopole field within the context of NED. The nonlinear Lagrangian in the present case can be expressed in a non-polynomial form of the Maxwell invariant. Such a Lagrangian does not admit a linear Maxwell limit. By employing the spacetime of Hayward and different equations of state of generic form, $p=\psi(\sigma)$, on the thin shell we plot possible stable regions. Amongst these, linear, logarithmic, and different Chaplygin gas forms are used, and stable regions are displayed. The method of identifying these regions relies on the reduction of the perturbation equations to a harmonic equation of the form $\ddot{x}+\frac{1}{2} V^{\prime \prime}\left(a_{0}\right) x=0$ for $x=a-a_{0}$. Stability simply amounts to the condition $V^{\prime \prime}\left(a_{0}\right)>0$, which is plotted numerically. In all different equations of state we obtained stable regions and observed that the Hayward parameter $\ell$ plays a crucial role in establishing the stability. That is, for higher $\ell$ value we have enlargement in the stable region. The trivial case, $\ell=0$, corresponds to the Schwarzschild case and is well known. We have considered also perturbations with small velocity. It turns out that our TSW is no more stable against such a kind of perturbations. We would like to add here that a stable spherically symmetric wormhole in general relativity has been introduced in [47]. Finally, we admit that in each case our energy density happens to be negative so that we are confronted with exotic matter. In a separate study we have shown that not to have exotic matter to thread the wormhole we have to abandon spherical symmetry and consider prolate/oblate spheroidal sources [48].

Open Access This article is distributed under the terms of the Creative Commons Attribution License which permits any use, distribution, and reproduction in any medium, provided the original author(s) and the source are credited.

Funded by $\mathrm{SCOAP}^{3}$ / License Version CC BY 4.0.

\section{References}

1. M. Visser, Phys. Rev. D 39, 3182 (1989)

2. M. Visser, Nucl. Phys. B 328, 203 (1989)

3. P.R. Brady, J. Louko, E. Poisson, Phys. Rev. D 44, 1891 (1991)

4. E. Poisson, M. Visser, Phys. Rev. D 52, 7318 (1995)

5. M. Ishak, K. Lake, Phys. Rev. D 65, 044011 (2002)
6. C. Simeone, Int. J. Mod. Phys. D 21, 1250015 (2012)

7. E.F. Eiroa, C. Simeone, Phys. Rev. D 82, 084039 (2010)

8. F.S. Lobo, Phys. Rev. D 71, 124022 (2005)

9. E.F. Eiroa, C. Simeone, Phys. Rev. D 71, 127501 (2005)

10. E.F. Eiroa, Phys. Rev. D 78, 024018 (2008)

11. F.S.N. Lobo, P. Crawford, Class. Quantum Grav. 22, 4869 (2005)

12. N.M. Garcia, F.S.N. Lobo, M. Visser, Phys. Rev. D 86, 044026 (2012)

13. S.H. Mazharimousavi, M. Halilsoy, Z. Amirabi, Phys. Lett. A 375, 3649 (2011)

14. M. Sharif, M. Azam, Eur. Phys. J. C 73, 2407 (2013)

15. M. Sharif, M. Azam, Eur. Phys. J. C 73, 2554 (2013)

16. S. H. Mazharimousavi, M. Halilsoy, Eur. Phys. J. C 73, 2527 (2013)

17. G.A.S. Dias, J.P.S. Lemos, Phys. Rev. D 82, 084023 (2010)

18. J.P.S. Lemos, F.S.N. Lobo, S.Q. Oliveira, Phys. Rev. D 68, 064004 (2003)

19. J. Bardeen, Proceedings of GR5, Tiflis, U.S.S.R. (1968)

20. A. Borde, Phys. Rev. D 50, 3392 (1994)

21. A. Borde, Phys. Rev. D 55, 7615 (1997)

22. E. Ayon-Beato, A. Garcia, Phys. Rev. Lett. 80, 5056 (1998)

23. K. Bronnikov, Phys. Rev. Lett. 85, 4641 (2000)

24. K. Bronnikov, Phys. Rev. D 63, 044005 (2001)

25. K.A. Bronnikov, V.N. Melnikov, G.N. Shikin, K.P. Staniukovich, Ann. Phys. (USA) 118, 84 (1979)

26. S.A. Hayward, Phys. Rev. Lett. 96, 031103 (2006)

27. W. Israel, Nuovo Cimento 44B, 1 (1966)

28. V. de la Cruzand, W. Israel, Nuovo Cimento 51A, 774 (1967)

29. J.E. Chase, Nuovo Cimento 67B, 136 (1970)

30. S.K. Blau, E.I. Guendelman, A.H. Guth, Phys. Rev. D 35, 1747 (1987)

31. R. Balbinot, E. Poisson, Phys. Rev. D 41, 395 (1990)

32. M.G. Richarte, C. Simeone, Phys. Rev. D 80, 104033 (2009)

33. M.G. Richarte, Phys. Rev. D 82, 044021 (2010)

34. E.F. Eiroa, C. Simeone, Phys. Rev. D 76, 024021 (2007)

35. F.S.N. Lobo, Phys. Rev. D 73, 064028 (2006)

36. V. Gorini, U. Moschella, A.Y. Kamenshchik, V. Pasquier, A.A. Starobinsky, Phys. Rev. D 78, 064064 (2008)

37. V. Gorini, A.Y. Kamenshchik, U. Moschella, O.F. Piattella, A.A. Starobinsky, Phys. Rev. D 80, 104038 (2009)

38. E.F. Eiroa, Phys. Rev. D 80, 044033 (2009)

39. C. Bejarano, E.F. Eiroa, Phys. Rev. D 84, 064043 (2011)

40. E.F. Eiroa, G.F. Aguirre, Eur. Phys. J. C 72, 2240 (2012)

41. A.Y. Kamenshchik, U. Moschella, V. Pasquier, Phys. Lett. B 487, $7(2000)$

42. L.P. Chimento, Phys. Rev. D 69, 123517 (2004)

43. M. Sharif, M. Azam, JCAP 05, 25 (2013)

44. M. Jamil, M.U. Farooq, M.A. Rashid, Eur. Phys. J. C 59, 907 (2009)

45. C. Bambi, L. Modesto, Phys. Lett. B 721, 329 (2013)

46. Z. Amirabi, M. Halilsoy, S.H. Mazharimousavi, Phys. Rev. D 88, 124023 (2013)

47. K.A. Bronnikov, L.N. Lipatova, I.D. Novikov, A.A. Shatskiy, Grav. Cosmol. 19, 269 (2013)

48. S.H. Mazharimousavi, M. Halilsoy, Thin-shell wormholes supported by normal matter. arXiv:1311.6697

49. M.G. Richarte, Phys. Rev. D 88, 027507 (2013)

50. E.F. Eiroa, C. Simone, Phys. Rev. D 70, 044008 (2004)

51. C. Bejarano, E.F. Eiroa, C. Simeone, Phys. Rev. D 75, 027501 (2007)

52. E.F. Eiroa, C. Simeone, Phys. Rev. D 81, 084022 (2010)

53. M.G. Richarte, C. Simeone, Phys. Rev. D 79, 127502 (2009)

54. E. Rubín de Celis, O.P. Santillán, C. Simeone, Phys. Rev. D 86, 124009 (2012)

55. M. Sharif, M. Azam, J. Cosmol. Astropart. Phys. 04, 023 (2013) 